and German equivalents. The equivalents are also separately and alphabetically listed in thumb-indexed sections and are numerically keyed to the basic table. A Russian supplement is planned to appear in due course. There are very few physies periodicals in Dutch, and perhaps it would have been more useful to have omitted Dutch and included Russian in the original volume.

The compiler, W. E. Clason, must be congratulated on the clarity and conciseness of his definitions of the physical terms. It is not an easy task by any means to condense into a single sentence of not more than thirty words a precise statement of the meaning of some physical terms. There are naturally one or two lapses, for example, "photoelasticity" where Mr. Clason allows a personal feeling to creep in by saying this is a badly chosen term, and "crystal growth" where the statement "The direct growth of an ideal perfect crystal is almost impossible, except at enormously high supersaturations, because of the difficulty of nucleating a new surface on a completed surface of a crystal" can scarcely be regarded as a definition. There are a few misprints. Lennard-Jones is throughout referred to as Lerrard-Jones; in term 1,855 it should be the 'Lorenz number' not 'Lorentz number'; and the Nusselt number is usually abbreviated to $\mathrm{Nu}$ not $\mathrm{N} \mu$.

The system of thumb indexing provided gives easy and quick access to a particular language and there is a tape bookmark. For a dictionary, which has to be handled a great deal and rather more roughly than most books, the binding which is claimed by the publishers to be flexible is by no means strong enough. The titlepages of my own copy when received had already parted company from the binding.

S. WeINTROUB

\section{LOW TEMPERATURES AND OTHER TOPICS}

\section{Low-Temperature Physics}

Conduction Electrons-Superconductivity-HeliumMagnetism--Mössbauer Effect-Defects and Irradiation. Edited by C. DeWitt, B. Dreyfus and P. G. de Gennes. (Lectures delivered at Les Houches during the 1961 Session of the Summer School of Theoretical Physics, University of Grenoble.) Pp. xvi+638. (New York: Gordon and Breach, Science Publishers, Inc., 1962.) 20 dollars, cloth; Academic and Students edition (paper) 9.50 dollars.

CUMMER schools in physics have become very fashion$\checkmark$ able in the past few years, and each one is inevitably followed by the appearance of a book containing the substance of the lectures delivered. These are now multiplying at such a rate that in some fields they threaten to exceed in total bulk the original papers they are meant to survey. But the summer schools held at Les Houches have a longer history than most, and their published lectures enjoy a deservedly high reputation, which is comfortably sustained by the present volume. It has the added merit that one or two of the topics discussed in it have not been previously surveyed, at least within the past year or two.

The following list summarizes the contents of the book by topic, author, length in pages and language ( $E$ or $F$ : English or French): electrons in metals (Pippard, 143, $E$ ); superconductivity (Tinkham, 81, E); magnetism and superconductivity (Suhl, 27, E); liquid helium three and four (Beenakker, $41, E$ ); co-operative aspects of magnetism (Herpin, 100, $F^{\prime}$ ); small-grain antiferromagnetism (Néel, 30, $F$ ); spin waves (Kittel, 37, $E$ ); Mössbauer effect (Abragam, 70, $F^{\prime}$ ); and point defects and radiation damage (Friedel, 76, F). Thus just more than half the book is in English and the rest in French, and the reader will need to be reasonably fluent in both languages to derive full value from it.
Only a quarter of the book is devoted to the classic low. temperature problems of superconductivity and liquid helium; the remainder is concerned with problems in which low temperatures are used as a tool of research, and sometimes only a minor tool. Indeed, Friedel dis. armingly opens his survey by remarking that " ... the phenomena of interest often occur at high temperatures. ... The study of quenching and of irradiation at low temperatures is only a pretext for including this course in this year's programme". In fact a more appropriate title would have been 'Some Topics in Low-temperature and Solid-state Physics'.

With this reservation, the book can be warmly recommended to anyone interested in these topics. There is no need to review the individual contributions: the list of authors is sufficient guarantee of their quality. But the complete absence of references from some of the articles seriously diminishes their usefulness for the wider audience to whom they are now offered.

Lastly, the reader must be warned that the book was printed in Hungary, and is fairly liberally sprinkled with misprints, not all of them trivial; and that the paperbound edition (available to workers in the field, on production of bona fides) will disintegrate fairly rapidly if it is used as much as it deserves to be. R. G. CHAMBers

\section{X-RAY ABSORPTION ANALYSIS IN BIOLOGY}

\section{$\mathrm{X}$-ray Microanalysis in Biology and Medicine}

By Prof. Arne Engström. (Elsevier Monographs: Biophysies Section.) Pp. viii + 92. (Amsterdam: Elsevier Publishing Company, 1962.) $20 s$.

HE development of quantitative methods of microradiography has been largely the work of Engström and his school at the Karolinska Institut, Stockholm. This monograph, based on lectures given by him at the University of California, provides the first systematic account of this type of X-ray absorption analysis, with some mention also of the emission and diffraction techniques. The first two chapters describe the chief properties of X-rays and the various methods of forming images with them. Chapters 3 and 4 give details of the experimental procedures for microradiography with X-rays of medium-to-hard and ultra-soft wave-lengths respectively, the examples chosen for discussion being bone and tissue sections. Chapter 5 is concerned with the quantitative determination of the dry mass and water content of tissues by microdensitometry of an X-ray negative of the sample and a reference system. The results obtained from different tissues are briefly indicated, with references to the original papers. Elementary analysis by X-ray absorption is described in Chapter 6, with particular reference to the determination of calcium and phosphorus in bone and of sulphur in the skin. Chapters 7 and 8 give short accounts of the methods of X-ray emission microanalysis and microdiffraction respectively. The book ends with a list of references for further reading and with a short subject index. It is aimed at research workers in biology and medicine, particularly in the field of cytology, who are likely to be faced with the problem of estimating the dry mass of cells or the inorganic elements in them. The treatment is elementary, but clear and thorough. The required mathematics is carefully introduced and well set out, and should be readily followed by any biologist who is familiar with analytical procedures, though he may initially need a little help in how to handle exponentials when it comes to working out results. The text is illustrated with 36 line-diagrams and supplemented by 12 tables of data. Altogether it is a most satisfactory introduction to a set of techniques which as yet are not very well known to biologists.

V. E. Cosslett 